BMJ Open Sport \& Exercise Medicine

\title{
Use of continuous glucose monitoring for sport in type 1 diabetes
}

\author{
Alif Abdulrahman, ${ }^{1}$ Janisha Manhas, ${ }^{1}$ Hannah Linane, ${ }^{2}$ Mark Gurney, ${ }^{2}$ \\ Catriona Fitzgerald, ${ }^{1}$ Esther O'Sullivan ${ }^{1,2}$
}

To cite: Abdulrahman A, Manhas J, Linane $\mathrm{H}$, et al. Use of continuous glucose monitoring for sport in type 1 diabetes. BMJ Open Sport \& Exercise Medicine 2018;4:e000432. doi:10.1136/ bmjsem-2018-000432

Accepted 12 November 2018

Check for updates

\section{C) Author(s) (or their} employer(s)) 2018. Re-use permitted under CC BY-NC. No commercial re-use. See rights and permissions. Published by BMJ.

${ }^{1}$ School of Medicine, National University of Ireland, Galway, Ireland

${ }^{2}$ Department of Diabetes, Endocrinology and Metabolism, Galway University Hospital, Galway, Ireland

Correspondence to Dr Esther 0'Sullivan; esther. osullivan2@hse.ie

\section{ABSTRACT}

Background The benefits of exercise for patients with type 1 diabetes (T1D) are difficult to balance with associated glycaemic excursions. The aim of this cohort study was to show that continuous glucose monitoring (CGM) could reduce glycaemic excursions in patients with T1D already using insulin pumps, exercising at moderate to high intensity.

Methods Questionnaires were used to identify patients with T1D using insulin pumps and naive to CGM use, who reported regular exercise. Six were enrolled and trained on Enlite sensor use with Medtronic Minimed Paradigm Veo system and given activity trackers and written advice on adjustment of insulin or carbohydrate intake for exercise. Resting heart rate (HR) and age were used to determine HR surrogates of moderate and high-intensity exercise. They were to exercise as usual for 3 weeks (run-in week, week 1 and week 2) using the activity trackers and heart rate monitors. Problem areas in Diabetes, Hypoglycaemia fear survey II, Diabetes Technology Questionnaire and Gold scores were completed prior to run-in and at the end. The downloaded sensor glucose data were used to compare the change in time in range (glucose $3.9-10.0 \mathrm{mmol} / \mathrm{L}$ ) from week 1 to week 2.

Results For the duration of exercise, this time in glucose range increased from $72 \pm 20$ to $88 \% \pm 16 \%, p=0.05$. The time in hypoglycaemia range (glucose $<3.9 \mathrm{mmol} / \mathrm{L}$ ) went from $3.9 \pm 7.9$ to $2.4 \% \pm 4.8 \%, p=0.39$. The time in hyperglycaemia range $(>10 \mathrm{mmo} / \mathrm{L}$ ) reduced from $24 \pm 19$ to $10 \% \pm 17 \%, p=0.04$.

Conclusion These results demonstrate the benefit of CGM use for patients with T1DM doing moderate-intensity to high-intensity exercise.

\section{INTRODUCTION}

Exercise is recommended to patients with type 1 diabetes (T1D) to improve their cardiovascular health and reduce their risk factors for cardiovascular disease. ${ }^{1}$ However, exercise, especially moderate-intensity to high-intensity exercise, can lead to excursions in glucose levels that are difficult to manage. ${ }^{2}$ Education about appropriate insulin dose adjustments for different types of exercise can be very helpful, but is not always enough to eliminate this problem. ${ }^{3}$ The use of insulin pump therapy instead of multiple daily injections (MDI) can also help ${ }^{4}$
Key messages

What are the new findings?

- The use of continuous glucose monitoring (CGM) for selected people already using insulin pumps to manage type 1 diabetes (T1D) can improve the time glucose stays in target during exercise.

How might it impact on clinical practice in the near future?

- It will become more relevant in the future if these devices become more readily available and more people with T1D have access to them.

- This finding is relevant to clinical practice now as it shows that people with T1D who exercise at moderate to high intensity should be considered for CGM use.

but even patients using this form of insulin delivery, where the insulin basal rate can be adjusted readily, often continue to struggle to maintain good glycaemic control around the time of exercise. ${ }^{56}$ Nocturnal hypoglycaemia after aerobic exercise sessions is a typical problem, ${ }^{78}$ but hypoglycaemia and hyperglycaemia during or immediately after exercise also occur. ${ }^{9}$ Hyperglycaemia is more likely to occur after high-intensity or anaerobic exercise. ${ }^{10}$ Patients sometimes target hyperglycaemia before exercise as a mechanism of avoiding hypoglycaemia during it. A useful tactic to avoid hypoglycaemia during exercise is to intersperse a $10 \mathrm{~s}$ sprint into a lower-intensity aerobic exercise session. ${ }^{11}$ Studies in controlled settings have demonstrated the benefit of using real-time continuous glucose monitoring (CGM) to inform insulin and carbohydrate adjustments to avoid wide glycaemic excursions. ${ }^{12} 13$ The aim of this study was to examine the benefit of CGM for patients involved in regular moderate-intensity to high-intensity exercise in the real-life setting. This is a pilot study in a small group of people with T1D, the results of which will be used to inform a larger follow-on study. We feel further study in the area is important to 
inform how to best manage glycaemic excursions associated with exercise in this population.

\section{METHODS}

\section{Subjects}

Questionnaires were sent to all patients with T1D using insulin pumps attending the Diabetes Clinic at Galway University Hospital. Those naive to real-time CGM use who reported regular (at least twice per week) moderate-intensity to high-intensity exercise were invited to participate. Only patients who had used CGM in the past, who reported less exercise than this and inability to attend for study visits were excluded. Six were enrolled and trained on Enlite sensor use with Medtronic Minimed Paradigm Veo system. All were given written general advice on adjustment of insulin or carbohydrate intake, for different exercise intensities, duration and proximity to mealtime, and nutritional advice for exercise (table 1) (this is adapted from Exercise management in type 1 diabetes: a consensus statement). ${ }^{14}$ These instructions were used as guidelines only and insulin and nutritional requirement vary between individuals and also within the same individuals depending on their level of fitness. This was explained to the participants.

They were also given Polar ProTrainer 5 watches and shown how to use them with the accompanying heart rate (HR) monitor chest strap, to monitor their HR during exercise sessions. We measured their resting $\mathrm{HR}$, and together with age this was used to calculate the age-predicted maximum HR (220-age=HRmax). Using the Karvonen method we determined heart rate reserve (HRR=HRmax - HRrest). HR ranges $40 \%-70 \%$ and $70 \%-80 \%$ of HRR added to HRrest were used as surrogates of moderate-intensity and high-intensity exercise, respectively. Weight, body mass index and blood pressure were recorded at baseline, and a blood sample was sent to test Hba1c (IFCC assay) (table 2). They were instructed to exercise as usual for 3 weeks, turning the Polar watch on for the duration of exercise. The Moves app was installed on their own smartphones that they were asked to carry always to monitor their level of activity at times when they were not exercising; this app has been validated as a reliable activity tracker. ${ }^{15}$ It was used to give an indication of any exertion outside of the reported exercise sessions falling in to the category of exercise to be included in the analysis. At the end the run-in (week -1), subjects were contacted to troubleshoot any calibration or sensor change problems. If they consistently wore the sensor, exercised at least twice per week and used the Polar watch for exercise during the run-in week they were included in the study. Two subjects were not included. In one case, this was because regular exercise was not carried out due to an injury, in the other case it was because there was difficulty using the Polar watch and HR monitor for exercise sessions which involved Rugby training.

Problem areas in Diabetes (PAID),${ }^{16}$ Hypoglycaemia fear survey II (HFSII $)^{17}$ and Diabetes Technology Questionnaire $(\mathrm{DTQ})^{18}$ were completed prior to run-in and at

Table 1 Insulin and carbohydrate adjustment guidelines

Pre-exercise blood
glucose (mmol/L)
(target $7-10 \mathrm{mmol} / \mathrm{l})$

Action

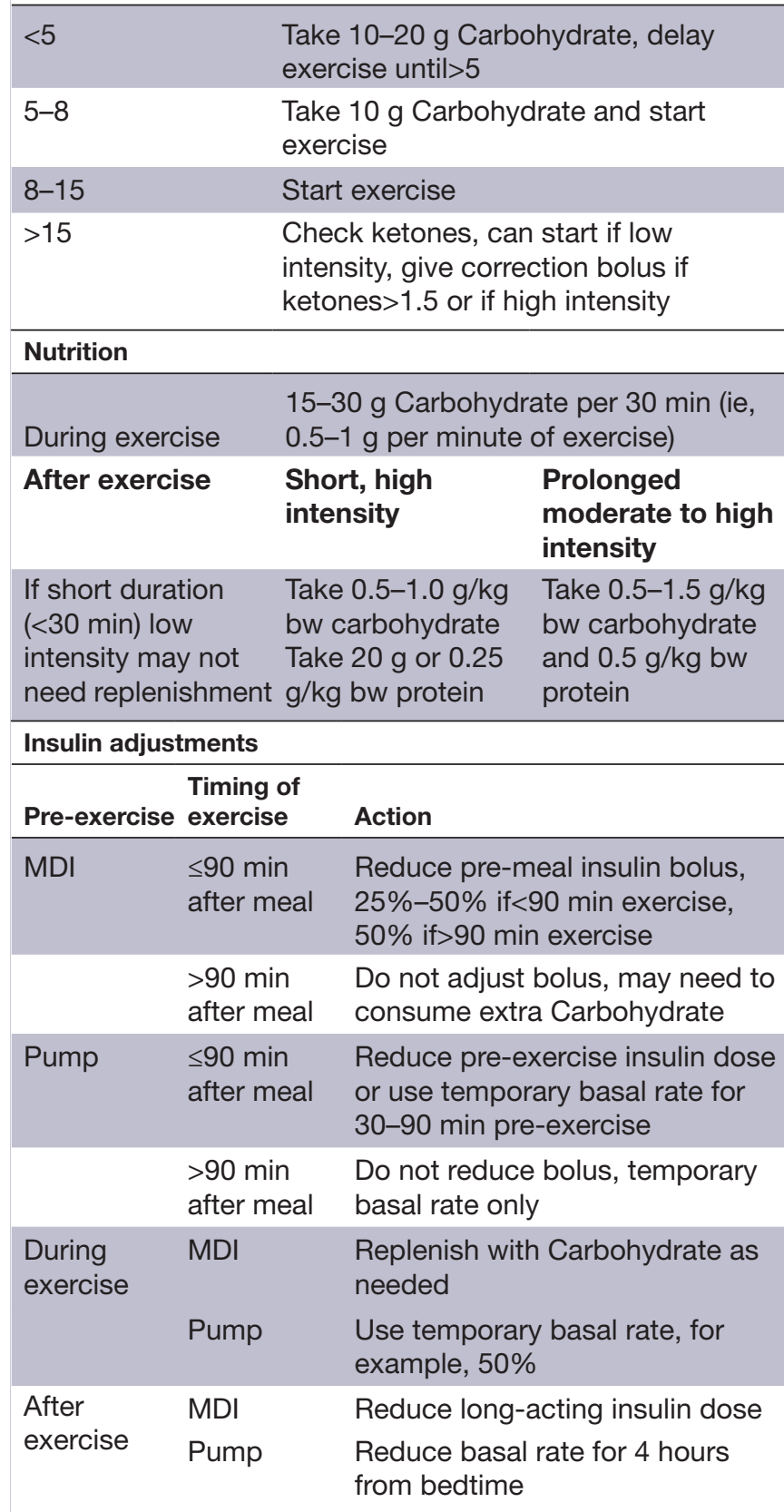

Continued 


\begin{tabular}{l} 
Table 1 Continued \\
\hline Insulin adjustments \\
\hline Timing of \\
Pre-exercise exercise Action \\
\hline
\end{tabular}

bw, body weight;

Additional recommendations: If first-time exercise, there is a prolonged hypoglycaemia risk so the basal rate should be reduced for the entire night using temporary basal rate setting; if on MDI the long -acting dose should be reduced that day/ night. Hypoglycaemia risk is also higher if exercise is carried out on sequential days, or there was a hypoglycaemic event on the day preceding exercise. Alcohol also increases the risk of hypoglycaemia. If hyperglycaemia is encountered post-exercise (especially likely if moderate-intensity/high -intensity exercise at the anaerobic/lactate threshold), a cool-down over 20-30 min will reduce the need to give a correction bolus. A $10 \mathrm{~s}$ sprint done before and/or during low/moderate -intensity exercise will elevate glucose levels and reduce the risk of hypoglycaemia during or after exercise.

the end of the study and the Gold score ${ }^{19}$ was obtained from each individual before the study to assess their level of hypoglycaemia unawareness. (This is a simple score from 1 to 7 in response to the question: Do you know when your hypos are commencing? Where 1 is where you are always aware, and 7 means you are never aware.) These are all validated questionnaires designed to quantify quality of life (QOL) and hypoglycaemia unawareness and changes in these parameters over time.

\section{Statistical analysis}

The downloaded sensor glucose data on Carelink Personal (Medtronic software for patient and health professional review of insulin and glucose data) were analysed in SPSS. Thresholds for glucose levels were set as follows: target range $3.9-10 \mathrm{mmol} / \mathrm{L}$, hypoglycaemia range $<3.9 \mathrm{mmol} / \mathrm{L}$ and hyperglycaemia range $>10$ $\mathrm{mmol} / \mathrm{L}$. All available sensor glucose values were included in the analysis (one value is provided every 5 min while the sensors are worn). There was no significant difference in the number of missing glucose values between subjects. The periods of time the patients spent exercising were determined from the HR monitor, where glucose data associated with an HR at least $0.4 \times$ (HRmax) was included. Student's paired t-test was used to compare time in target range, in week 1 to week 2.

\section{Table 2 Baseline demographics}

\begin{tabular}{ll}
\hline & Mean (SD) \\
\hline Subjects, $\mathrm{n}$ & 4 \\
Age (SD) & $38(6)$ \\
Male & 4 \\
Duration of diabetes (in years) & $21(9)$ \\
Body mass index $\left(\mathrm{kg} / \mathrm{m}^{2}\right)$ & $28(3)$ \\
HbA1c $(\mathrm{mmol} / \mathrm{L})$ & $58(9)$ \\
\hline
\end{tabular}

\section{RESULTS}

Three of the subjects wore sensors for all 21 days of the study, one wore it for 19 days. The percentage of missing data points did not differ significantly from week to week and duplicates were removed. There was an increase in the time in the target glucose range during exercise, in week 2 compared with week $1(72.4 \% \pm 20 \%$ to $88.1 \% \pm 15.6 \% \mathrm{p}=0.05$ ), and a reduction in the time in the hyperglycaemia range $(24 \% \pm 19 \%$ to $10 \% \pm 17 \% \mathrm{p}=0.04)$ (figure 1B). There was no significant reduction in hypoglycaemia; however, the improvement in the time in target is not all attributable to reduction in the time in the hyperglycaemia range-the mean time in the hypoglycaemia during exercise reduced from $3.9 \% \pm 7.9 \%$ to $2.4 \% \pm 4.8 \%, \mathrm{p}=0.39$. When the sensor glucose data for the entire 2-week period were evaluated, there was no significant difference from week 1 to week 2 (figure 1A), or for the time from 1 hour before to 4 hours after exercise (figure 1C).

The Gold score ranged from 1 to 4 indicating no hypoglycaemia unawareness in one individual and a mild to moderate degree in the other subjects. The other QOL measures showed low levels of anxiety (HFS II Worry scale scores were $13 \pm 16$ out of a possible $0-72$ ) and behaviour due to fear of hypoglycaemia $(14 \pm 7$ out of a possible $0-48)$. The PAID scores were also moderate $(16 \pm 14$ out of a possible 0-80) (table 3). Nonetheless, all of the QOL scores showed a non-significant trend towards improving following use of the CGM for the duration of the study.

\section{DISCUSSION}

This small real-life study demonstrates the potential benefit of using CGM for exercise. We showed that there was a significant improvement in the time that interstitial glucose was in the target range $(3.9-10 \mathrm{mmol} / \mathrm{L})$ of $16 \%$ during exercise. This occurred over just 2 weeks, and we feel this improvement translates into a clinically significant benefit for these patients. They were involved in a variety of types of exercise, cycling, running, soccer and weightlifting and all had improvements in their glycaemic control during exercise between week 1 and week 2 of the study. Although there was no significant change in the time in the hypoglycaemia range from week 1 to week 2, there was a trend in this direction, and the time spent in the hypoglycaemia range was small. This difference did not reach significance as it was not powered to do so due to the small number of subjects. The same reason can explain the lack of difference in the time in target range overall from week 1 to week 2 (including time exercising and not), where time in the hypoglycaemia reduced from $5.6 \% \pm 3.1 \%$ to $3.4 \% \pm 1.2 \%, \mathrm{p}=0.15$, and hyperglycaemia time reduced from $29 \% \pm 16 \%$ to $25 \% \pm 15 \%, \mathrm{p}=0.11$. Similar results were found for the time from 1 hour before and exercise session started to 4 hours afterward. This is a period that carries a high risk for wide glycaemic excursions; we expect a larger study would show significant reductions in time in the hypoglycaemia and hyperglycaemia ranges during this 


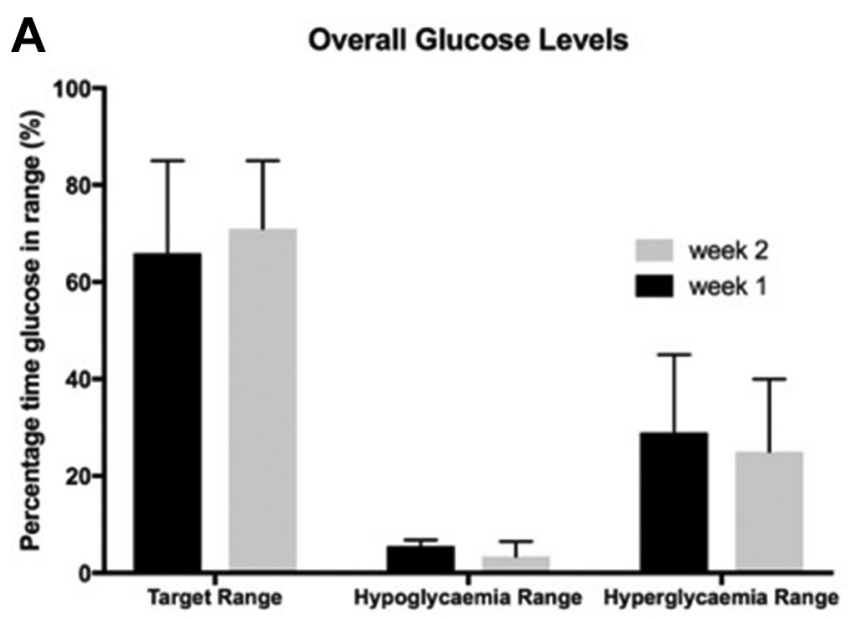

B Glucose levels during exercise
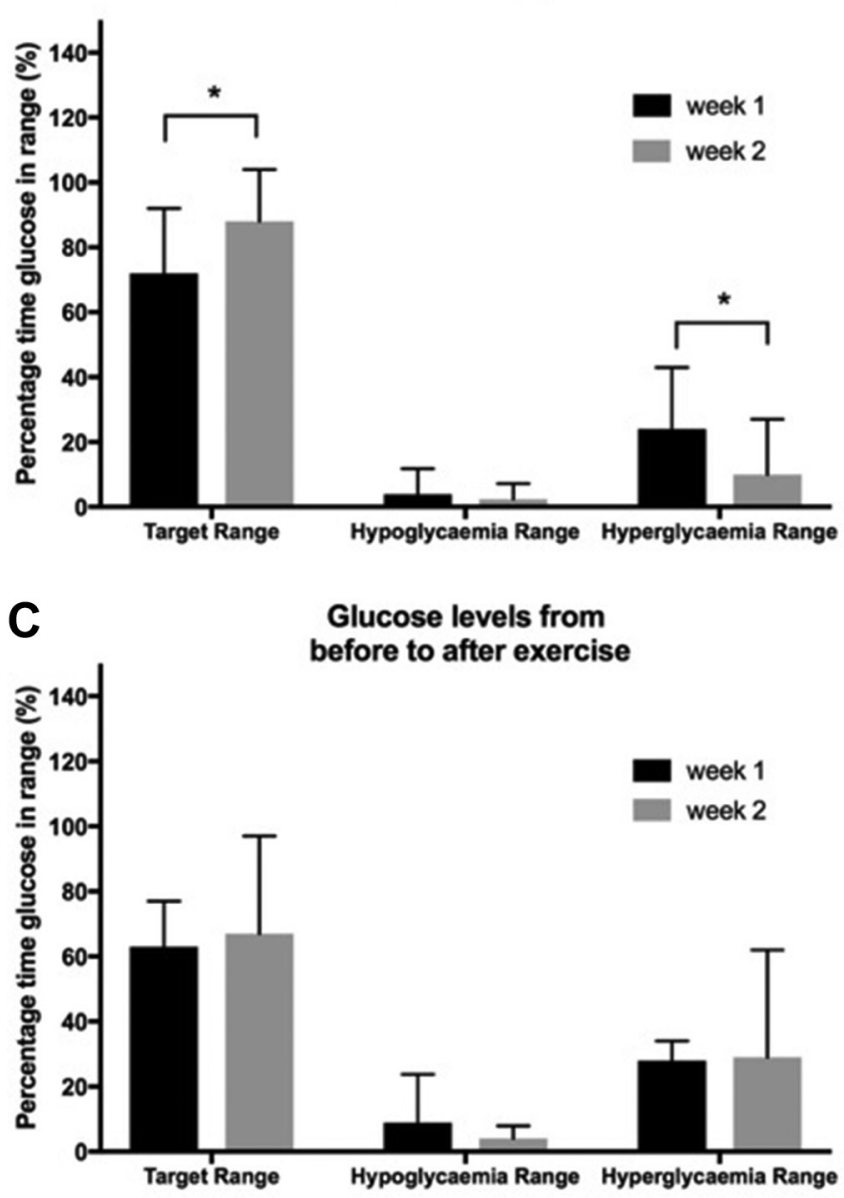

Figure 1 The sensor glucose values were binned into time in target, time in hypoglycaemia range and time in hyperglycaemia range and are shown as percentage in each range. (A) All available glucose values over the 2 weeks for the study are divided into the three different ranges and week 1 is compared with week 2.(B) The glucose values that correspond to the time of exercise (determined from the polar watch heart rate $(\mathrm{HR})$ data (all time that $\mathrm{HR}+\mathrm{HRR}$ $>40 \% \mathrm{HRmax}$ ) was divided into the three target ranges and compared between week 1 and week 2. (C) The glucose values from 1 hour before to 4 hours after exercise.

\begin{tabular}{|c|c|c|c|}
\hline Questionnaire & $\begin{array}{l}\text { Before study } \\
\text { score }\end{array}$ & $\begin{array}{l}\text { At end of } \\
\text { study score }\end{array}$ & $\begin{array}{l}\text { Change in } \\
\text { score }\end{array}$ \\
\hline PAID & $16 \pm 14$ & $15 \pm 11$ & $-1.5 \pm 6$ \\
\hline $\begin{array}{l}\text { HFS II } \\
\text { behaviour }\end{array}$ & $14 \pm 7$ & $9 \pm 2$ & $-5.5 \pm 8$ \\
\hline HFS II worry & $13 \pm 16$ & $9 \pm 9$ & $-3.8 \pm 12$ \\
\hline
\end{tabular}

HFS, Hypoglycaemia fear survey II; PAID, Problem areas in Diabetes.

period. Our study showed a high proportion of time in hypoglycaemia during this time $(8.9 \% \pm 14.9 \%$ in week 1 , $4.1 \% \pm 3.8 \%$ in week 2 ).

The subjects used the CGM system for a run-in week, and there were no overall significant improvements demonstrated between that week and subsequent weeks, suggesting that these patients needed some time to get used to the device and how to adjust their insulin according to the CGM glucose data available. The only input from the diabetes research team was at the beginning of the study - when subjects were given basic instructions on insulin adjustment for exercise (table 1) and shown how to use the study devices. There was a further contact after the run-in week, in person or by phone, to troubleshoot any problems, but we did not review their data or provide individualised advice until the end of the study. Each subject had access to Carelink Personal and reviewed their data both in real time and retrospectively to help to inform insulin dose adjustment. All of the patients were already insulin pump users, so the benefits they gained demonstrate the role of CGM in addition to insulin pump therapy in the setting of exercise. HbA1cs varied from on target to significantly elevated indicating a wide range of glycaemic control at baseline.

The HFSII and PAID questionnaires show there was a moderate level of fear of hypoglycaemia and anxiety around management of their diabetes in general. The DTQ showed the most consistent responses at the end of the study were of a slight or great reduction in the stress around participating in sport and avoiding hypoglycaemia. All four found the CGM device user-friendly, for the two patients that did not pass the run-in phase CGM compliance was not the issue; it was due to inability to exercise and difficulty with the HR monitor and exercise watch.

\section{Potential benefits}

Technology for the management of diabetes continues to advance, and healthcare professionals involved in diabetes management are challenged with the question of which patients are likely to benefit from the use of CGM. As it stands CGM in the UK and Ireland is funded in cases where there is a specific need such as hypoglycaemia unawareness or recurrent hypoglycaemia. More research is needed to determine what other patients are likely to benefit not just in terms of HbAlc, but 
also in terms of reduction of glycaemic excursions and improvement in QOL measures. There is some evidence to suggest the amplitude of glycaemic excursions and not just $\mathrm{HbAlc}$ is correlated and positively associated with development of diabetes complications, but more longitudinal CGM-based studies are needed ${ }^{20}$ CGM use has the potential to reduce these excursions, thereby possibly reducing the risk of development or progression of these complications. There is some evidence to demonstrate the benefits to be gained in terms of QOL for patients who access CGM after previously relying on finger-stick tests for self-monitoring of blood glucose. ${ }^{21}$ Not all patients report reduced anxiety, but the continuous glucose data along with trend arrows, predictive alarms and low-glucose suspend features in some cases provide great reassurance and in cases of hypoglycaemia unawareness have the potential to avoid life-threatening hypoglycaemia. The average duration of diabetes in the group was considerable $(21 \pm 9$ years $)$, which may be associated with lower scores related to anxiety about their diabetes, and people with closer to the diagnosis of diabetes may show greater improvement in anxiety scores with the use of CGM. There are tools that help predict whether individual children with T1D will benefit from the use of CGM particularly in terms of consistent use. ${ }^{22}$ Further study is needed to determine how to predict the type of adults with T1D that will benefit.

\section{Limitations}

To date cost-effectiveness analyses have not shown clear indications for the use of these devices. ${ }^{23}$ The cost involved is not just that of the sensor enabled pump and sensors, but also the cost of the diabetes educator hours involved in training patients on how to use of these devices effectively. Here we have shown that a variety of insulin pump users can achieve benefits from the additional use of CGM with minimal input form the diabetes team. All of these patients had previously attended a structured education course so had a good baseline knowledge of how to adjust their insulin doses themselves. There also may be an element of selection bias and the individuals who agreed to participate may represent more a cohort with a high level of motivation. It is also possible that there was a bias towards improvement in the QOL scores as the study was unblinded. However, we feel this study demonstrates that a short trial of CGM use can quickly determine whether patients already using insulin pump therapy are likely to be compliant with CGM use. Those patients participating in regular moderate-intensity to high-intensity exercise are a cohort who can quickly benefit from the addition of CGM to improve their glucose time in the target range, and also improve their QOL.

\section{Future developments}

T1D has a major impact on an individual's lifestyle, including their ability to partake in exercise safely and enjoyably. Progress in technology for the treatment of diabetes makes this more attainable. CGM whether as an integrated sensor augmented pump system or standalone device is available for selected patients, but many more stand to benefit from it especially those involved in regular moderate-intensity to high-intensity exercise. New developments hold even more promise to improve the lives of these individuals. One study in the real-life setting to investigate the use of CGM along with an accelerometer and algorithm that incorporated the exercise intensity showed reduction in hypoglycaemia. ${ }^{24}$ The future incorporation of HR and other data into a closed loop device should further facilitate avoidance of glycaemic excursions around exercise. In the meantime, as we have shown in this small pilot study, sensor-enabled insulin pumps have the potential to allow these patients to exercise while reducing their glycaemic excursions and anxiety about hypoglycaemia.

Acknowledgements Dr Amir Shafat Department of Physiology NUI,G loaned the Polar ProTrainer 5 watches for this study.

Contributors All of the authors made a substantial contribution to the manuscript. AA was responsible for training the subjects on devise use. JM and CF analysed the data. MG and $\mathrm{HL}$ assisted in design of the study and devised the questionnaire used for recruitment. EOS oversaw the study and wrote the manuscript.

Funding This study was funded by Irish Endocrine Society, Summer Studentship for Catriona Fitzgerald.

Competing interests CF received a grant from the Irish Endocrine Society. OS reports personal fees from Medtronic, outside the submitted work.

Patient consent for publication Not required.

Ethics approval All procedures performed in studies involving human participants were in accordance with the ethical standards of GUH and with the 1964 Helsinki declaration and its later amendments or comparable ethical standards.

Provenance and peer review Not commissioned; internally peer reviewed. Data sharing statement The raw data can be shared on request.

Open access This is an open access article distributed in accordance with the Creative Commons Attribution Non Commercial (CC BY-NC 4.0) license, which permits others to distribute, remix, adapt, build upon this work non-commercially, and license their derivative works on different terms, provided the original work is properly cited, appropriate credit is given, any changes made indicated, and the use is non-commercial. See: http://creativecommons.org/licenses/by-nc/4.0/

\section{REFERENCES}

1. Yardley JE, Hay J, Abou-Setta AM, et al. A systematic review and meta-analysis of exercise interventions in adults with type 1 diabetes. Diabetes Res Clin Pract 2014;106:393-400.

2. García-García F, Kumareswaran K, Hovorka R, et al. Quantifying the acute changes in glucose with exercise in type 1 diabetes: a systematic review and meta-analysis. Sports Med 2015;45:587-99.

3. Rabasa-Lhoret R, Bourque J, Ducros F, et al. Guidelines for premeal insulin dose reduction for postprandial exercise of different intensities and durations in type 1 diabetic subjects treated intensively with a basal-bolus insulin regimen (ultralente-lispro). Diabetes Care 2001;24:625-30.

4. Yardley JE, Iscoe KE, Sigal RJ, et al. Insulin pump therapy is associated with less post-exercise hyperglycemia than multiple daily injections: an observational study of physically active type 1 diabetes patients. Diabetes Technol Ther 2013;15:84-8.

5. Zaharieva DP, Riddell MC. Prevention of exercise-associated dysglycemia: a case study-based approach. Diabetes Spectr 2015;28:55-62.

6. Franc S, Daoudi A, Pochat A, et al. Insulin-based strategies to prevent hypoglycaemia during and after exercise in adult patients with type 1 diabetes on pump therapy: the DIABRASPORT randomized study. Diabetes Obes Metab 2015;17:1150-7.

7. Campbell MD, Walker M, Bracken RM, et al. Insulin therapy and dietary adjustments to normalize glycemia and prevent nocturnal hypoglycemia after evening exercise in type 1 diabetes: 
a randomized controlled trial. BMJ Open Diabetes Res Care 2015;3:e000085

8. MacDonald MJ. Postexercise late-onset hypoglycemia in insulindependent diabetic patients. Diabetes Care 1987;10:584-8.

9. Campbell MD, Walker M, Trenell MI, et al. Metabolic implications when employing heavy pre- and post-exercise rapid-acting insulin reductions to prevent hypoglycaemia in type 1 diabetes patients: a randomised clinical trial. PLoS One 2014;9:e97143.

10. Shetty VB, Fournier PA, Davey RJ, et al. Effect of Exercise Intensity on Glucose Requirements to Maintain Euglycemia During Exercise in Type 1 Diabetes. J Clin Endocrinol Metab 2016;101:972-80.

11. Bussau VA, Ferreira LD, Jones TW, et al. The 10-s maximal sprint: a novel approach to counter an exercise-mediated fall in glycemia in individuals with type 1 diabetes. Diabetes Care 2006;29:601-6.

12. Riddell MC, Milliken J. Preventing exercise-induced hypoglycemia in type 1 diabetes using real-time continuous glucose monitoring and a new carbohydrate intake algorithm: an observational field study. Diabetes Technol Ther 2011;13:819-25.

13. Garg S, Brazg RL, Bailey TS, et al. Reduction in duration of hypoglycemia by automatic suspension of insulin delivery: the inclinic ASPIRE study. Diabetes Technol Ther 2012;14:205-9.

14. Polonsky WH, Anderson BJ, Lohrer PA, et al. Assessment of diabetes-related distress. Diabetes Care 1995;18:754-60.

15. Gonder-Frederick LA, Schmidt KM, Vajda KA, et al. Psychometric properties of the hypoglycemia fear survey-ii for adults with type 1 diabetes. Diabetes Care 2011;34:801-6.

16. Juvenile Diabetes Research Foundation Continuous Glucose Monitoring Study Group. Validation of measures of satisfaction with and impact of continuous and conventional glucose monitoring. Diabetes Technol Ther 2010;12:679-84.
17. Høi-Hansen T, Pedersen-Bjergaard U, Thorsteinsson B. Classification of hypoglycemia awareness in people with type 1 diabetes in clinical practice. J Diabetes Complications 2010;24:392-7.

18. Smith-Palmer J, Brändle M, Trevisan R, et al. Assessment of the association between glycemic variability and diabetes-related complications in type 1 and type 2 diabetes. Diabetes Res Clin Pract 2014; 105:273-84.

19. Kubiak T, Mann CG, Barnard KC, et al. Psychosocial Aspects of Continuous Glucose Monitoring: Connecting to the Patients Experience. J Diabetes Sci Technol 2016;10:859-63.

20. Neylon OM, Skinner TC, O'Connell MA, et al. A novel tool to predict youth who will show recommended usage of diabetes technologies. Pediatr Diabetes 2016;17:174-83.

21. Riemsma R, Corro Ramos I, Birnie R, et al. Integrated sensoraugmented pump therapy systems [the MiniMed $®$ Paradigm ${ }^{\mathrm{TM}}$ Veo system and the Vibe ${ }^{\text {TM }}$ and G4® PLATINUM CGM (continuous glucose monitoring) system] for managing blood glucose levels in type 1 diabetes: a systematic review and economic evaluation. Health Technol Assess 2016;20:1-252.

22. Stenerson M, Cameron F, Payne SR, et al. The impact of accelerometer use in exercise-associated hypoglycemia prevention in type 1 diabetes. J Diabetes Sci Technol 2015;9:80-5.

23. Riddell MC, Gallen IW, Smart CE, et al. Exercise management in type 1 diabetes: a consensus statement. Lancet Diabetes Endocrinol 2017;5:377-90.

24. Kooiman TJ, Dontje ML, Sprenger SR, et al. Reliability and validity of ten consumer activity trackers. BMC Sports Sci Med Rehabil 2015;7:24 